\title{
PROPORTION OF PHYSIOTHERAPY STUDENTS HAVING DRY EYE DISEASE
} DURING COVID-19 PANDEMIC

\section{Physiotherapy \\ Monica Mahesha Physiotherapy Intern, K.J. Somaiya College of Physiotherapy, Sion Mumbai.

Tanvi Patole* Assistant Professor, K J Somaiya College of Physiotherapy, Sion Mumbai. \\ *Corresponding Author}

\section{ABSTRACT}

Background: The coronavirus outbreak has significantly affected people's lives. As lockdown was imposed, media usage increased for academic and entertainment purposes. The increased usage of VDT has become a risk factor for developing dry eye disease.

Methodology: This cross-sectional study was conducted on undergraduate and postgraduate students. A validated questionnaire was distributed digitally and the severity of disease was measured using the Ocular Surface Disease Index (OSDI) questionnaire.

Conclusion: The occurrence of dry eye disease in Physiotherapy students with the age group 18-27 years was found to be 54.35\%. Thus, awareness about Dry Eye Disease (DED) and regular screening is required at regular intervals.

\section{KEYWORDS}

dry eye disease, visual display terminal, physiotherapy students, Ocular surface Disease Index (OSDI)

\section{INTRODUCTION}

In February 2020, the coronavirus outbreak was declared as a pandemic by the World Health Organization (WHO) ${ }^{1}$. The lockdown was ordered as a preventive measure. It affected several routines, work and mainly education. Many educational institutions and commercial establishments were shut down ${ }^{2}$. As lockdown was imposed, citizens spent more time on one or the other Visual Display Terminal (VDTs) for academic, social and recreational purposes. Use of VDT is one of the etiology of dry eye disease ${ }^{3,4}$.

Dry Eye Disease (DED) is defined as multifactorial disease of the tears and ocular surface that results in symptoms of discomfort, visual disturbance, and tear film instability with potential damage to the ocular surface 5 . The Symptoms of DED include ocular burning, foreign body sensation, stinging sensation, pain, photophobia and blurred vision ${ }^{5,15}$

Due to recent increase in the use of technology and digital devices, DED has become common mainly among young aged adults. During lockdown undergraduate and post-graduate students used digital platforms for learning including Physiotherapy students ${ }^{2}$. Therefore, this study was conducted to find the proportion of Physiotherapy students having DED during the COVID- 19 pandemic.

\section{METHODOLOGY:}

Approval of the study was taken from the institutional review board. The questionnaire was distributed digitally. The consent was taken and participants were asked to fill the demographic data such as age, gender and academic year. Further participants were questioned to find out the duration of exposure to VDTs use per day. The participants were directed to complete the Ocular Surface Disease Index (OSDI) ${ }^{6}$ questionnaire. A total of 230 completed questionnaires were received.

\section{OUTCOME MEASURES:}

The $\mathrm{OSDI}^{6}$ is a 12 -item questionnaire designed to assess symptoms seen in DED. The OSDI questionnaire has 3 subdomains: ocular symptoms, vision-related function, and environmental triggers. Participants have to rate their responses on a 0 to 4 scale, where 0 indicates "none of the time" and 4, "all of the time". The total OSDI score was then calculated based on the following formula: ([sum of scores for all answered questions] $\times 25) /([$ total number of answered questions]). Participants were then classified into four categories based on their total score: normal (scores: 0-12), mild (13-22), moderate $(23-32)$, and severe dry eye (33-100). The OSDI is a valid and reliable instrument for measuring the severity of DED.

\section{RESULTS:}

Demographic Details: Data collected was analysed using Microsoft Excel version 2010. The response rate was $74.43 \%$. Out of 230 respondents, $210(90.5 \%)$ were females and $20(9.5 \%)$ were males. The mean age of 230 participants included in this study was $22.5 \pm 2.87$ (18-27 years). The demographic details of participants are shown in Table 1.

Severity based on OSDI score: Based on OSDI score, participants were divided into normal, mild, moderate, and severe DED respectively. $45.65 \%(105 / 230)$ were found Normal and $54.35 \%$ $(125 / 230)$ showed symptoms of DED. Out of $54.35 \%(125 / 230)$, $37.6 \%(47 / 125)$ had mild, $27.2 \%(34 / 125)$ had moderate and $35.2 \%$ (44/125) had severe DED (table 1)

Dry eye disease by academic year: In this study, the data was collected academic year wise. Out of 230 participants, $52.27 \%$ from the first year of Bachelor of Physiotherapy (BPTh), 58.82\% from II BPTh, $50 \%$ from III BPTh and $48.84 \%$ students from IV BPTh showed symptoms of DED. It was observed that nearly half $(52.9 \%)$ of the participants in undergraduate program had DED. $58.33 \%$ of students studying in the first year of Master of Physiotherapy (MPTh) and 75\% from second MPTh showed symptoms of DED (table 1)

Time of exposure to Video display terminal (VDT): The participants commonly used Smartphones (98.3\%), Laptop (58.7\%) and Television (52.6\%). 125 participants had DED and out of that $39.13 \%(9 / 23)$ had VDT use for $2-4$ hours. $48.84 \%$ (42/86) students had VDT exposure for 4-6 hours. $55.84 \%$ (43/77) students had it for 6-8 hours and $73.81 \%$ $(31 / 42)$ had $>8$ hours of VDT usage. Out of 125 participants who had DED, 116 participants used VDTs for more than 4 hours. (Figure 1)

Table 1: Demographic Details, Severity Of DED, OSDI Scores And DED According To Academic Year

\begin{tabular}{|l|l|}
\hline Variables & Data \\
\hline Age $($ Mean \pm SD) & $22.5 \pm 2.87$ \\
\hline Sex & Percentages/ Number of \\
Males & students \\
Females & $9.5 \%(20)$ \\
\hline Severity of DED & $90.5 \%(210)$ \\
Normal & Percentage/ Number of \\
Mild DED & $45.65 \%(105)$ \\
Moderate DED & $37.6 \%(47)$ \\
Severe DED & $27.2 \%(34)$ \\
\hline OSDI score (Severity of DED) & $35.2 \%(44)$ \\
Mild & Mean score \\
Moderate & 17.79 \\
Severe & 26.11 \\
\hline Academic year & 45.49 \\
& Number of students \\
I BPTh & $(\mathrm{DED} /$ Normal) \\
II BPTh & $46 / 42$ \\
III BPTh & $30 / 21$ \\
IV BPTh & $12 / 12$ \\
I MPT & $21 / 22$ \\
II MPT & $7 / 5$ \\
\hline
\end{tabular}

\section{DISCUSSION:}

This study was conducted to determine the proportion of young aged students $(22.5 \pm 2.87)$ having DED using the $\mathrm{OSDI}^{6}$ questionnaire. The 
occurrence of DED in this study data was found to be $54.35 \%$. That coincides with study conducted in North India. Titiyal et al conducted a cross-sectional observational study ${ }^{7}$ on students between the age group of 18 years to 27 years in North India. The participants of all age groups (above 10 years of age) were included in this study. The study concluded that the prevalence of DED was $32 \%$ and it was commonly seen in young aged adults. Patients who used VDT for 4 hours or more and with contact lens on had increased risk for developing DED.

\section{Time of exposure to VDT}



Figure 1: Time Of Exposure To VDT

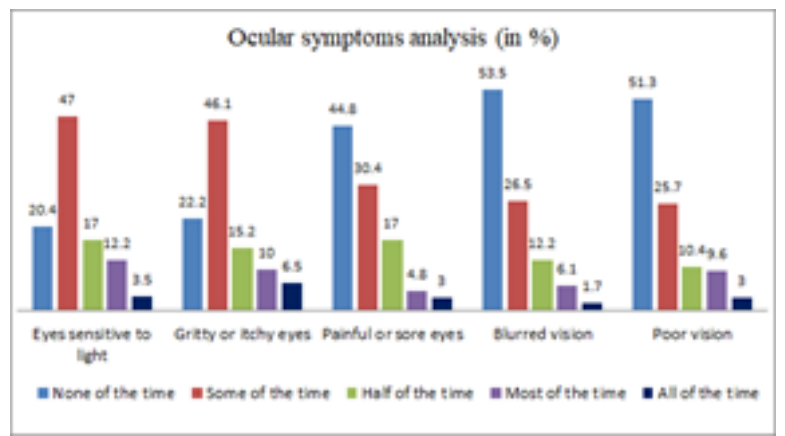

Figure 2: Ocular Symptoms Distribution In Participants

The use of VDTs such as smartphones, tablets, laptops, etc have significantly increased during the COVID- 19 times. Students are using various VDTs for attending online lectures, webinars, reading ebooks and for entertainment. Post-graduate students are delivering physical therapy via tele-rehabilitation. Thus, exposure time to VDTs could have increased. The high levels of concentration and cognition are required during VDT usage. Therefore, eye-blink rate decreases and the tear fluid evaporation increases. This disturbs homeostatic balance of the ocular surface system and leads to the symptoms of $\mathrm{DED}^{3}$. The increased use of VDT affects tear stability. Tear film stability depends on factors like blink reflex, healthy lacrimal tissue and tear film

In this study, students with more than 4 hours VDT usage showed increased risk of dry eye disease (figure 1). Kolla A et al ${ }^{10}$ conducted a study to assess prevalence of DED among college students between the age group of 18-25 years. It concluded that there is a significant risk of dry eye especially in students who use VDTs for more than 4 hours per day. This supports the results of the current study. LCD and LED screens emit large amounts of blue light. Studies ${ }^{3,10}$ have shown that excessive exposure to blue light affects the eye. It has an effect on the retina and results in glaucomatous changes in ganglion cells, damage in photoreceptors and predisposition to macular degeneration.

Visual disturbance is also a common symptom seen in patients with DED. It affects the quality of life $(\mathrm{QoL})$ and visual performance in patients. Studies ${ }^{11,12}$ reported that DED has an impact on a patient's QoL such as ability to perform certain activities that require continuous visual attention like reading and driving.

It is important to create awareness about DED among all who are using VDTs for working, learning and for entertainment purposes. Studies ${ }^{13,14}$ suggest that yoga practice helps in reduction of visual discomfort and some symptoms of DED. Shirley Telles et al conducted a 60 days intervention based study ${ }^{13}$. It concluded that yoga helps in reduction of the visual discomfort including dryness, irritation, burning, redness, photo-sensitivity and helps in relaxation of eye muscles. VDT induced
DED can be prevented with ergonomic changes. The practice of eye care habits and modification of lifestyle and activities can help in prevention or reduction in symptoms due to DED.

The study has limitations as follows: Further confirmation of DED could have been done by using clinical ophthalmological examinations. Risk factors such as use of contact lens, eye drops, Vitamin A deficiency, VDT usage at night, etc associated with DED were not considered in the study.

\section{CONCLUSION:}

The occurrence of DED in the study was found to be $54.35 \%$. Out of that $37.6 \%$ had mild, $27.2 \%$ had moderate and $35.2 \%$ had severe DED. Dry eye disease may also be present to a greater extent in the healthy young population. Therefore, awareness about DED and regular screening is mandatory.

\section{REFERENCES:}

[1] S. Udhaya Kumar et al. (2020) The Rise and Impact of COVID-19 in India. Volume 7 Article 250 doi: $10.3389 /$ fmed.2020.0025.

[2] Nanigopal Kapasiaa, et al. (2020) Impact of lockdown on learning status of undergraduate and postgraduate students during COVID-19 pandemic in West Bengal, India. Children and Youth Services Review 116 105194. doi.org/ 10.1016/j.childyouth. 2020.105194.

[3] Choi JH, LiY et al. (2018) The influences of smartphone use on the status of the tear film and ocular surface. PLoS ONE 13(10): e0206541.

[4] Ayman Mohammed Baabdullah et al. (2018) The association between Smartphone addiction and Dry eye Disease: A Cross-sectional study. Journal of Nature and Science of Medicine 10.4103/JNSM.JNSM 51 18

[5] Lemp MA, Foulks GA. The definition and classification of dry eye disease: report of the Definition and Classification Subcommittee of the International Dry Eye Workshop (2007). Ocul Surf. 2007;5(2):75-92

[6] Schiffman RM, Christianson MD, Jacobsen G, Hirsch JD, Reis BL. Reliability and validity of the Ocular Surface Disease Index. Arch Ophthalmol. 2000;118:615-621

77] Titiyal et al Prevalence and risk factors of dry eye disease in North India: Ocular Surface disease Index - based cross-sectional Hospital study Indian Journal of Ophthalmology 2018 10.4103/ijo.IJO $698 \quad 17$

[8] Andrea Turolla et al Musculoskeletal Physical Therapy During the COVID-19 Pandemic: Is Telerehabilitation the Answer? Published by Oxford University Press on behalf of American Physical Therapy Association DOI: 10.1093/ptj/pzaa093

[9] Deborah F. Sweeney et al Tear film stability: A review Experimental Eye Research 117 (2013)

[10] Andrea Kolla et al Prevalence of dry eye in college students at uparwara community in raipur International Journal of Community Medicine and Public Health 2019 Sep;6(9):3768-3770.

[11] Miki Uchino et al Dry Eye Disease: Impact on Quality of Life and Vision Cur Ophthalmol Rep.; 1(2): 51-57. doi:10.1007/s40135-013-0009-1.

[12] Nina Asrini Noor Dry Eye Disease: The Undervalued Impact on Quality of Life W J Opthalmol \& Vision Res. 1(1): 2018. WJOVR. MS.ID.000505. DOI: 10.33552/WJOVR.2018.01.000505.

[13] Shirley Telles et al Effect of yoga on self-rated visual discomfort in computer users Head \& Face Medicine 2006, 2:46 doi:10.1186/1746-160X-2-46

[14] Dr.Sonali Pandey Tripathi et al Effects of Yoga on Discomfortness of Vision in Regula Users of Computer in Population of Western Uttar Pradesh. IJSR Volume : 2 | Issue : 12 December 2013 ISSN No 2277 - 8179

[15] Makateb, A., and H. Torabifard. Dry eye signs and symptoms in night-time workers. J. Curr. Ophthalmol. 2017;29:270-273 